\title{
Simulation of the dynamic behavior of a rotor subject to base motion under variable rotational speed
}

\author{
Ryad Bouziani ${ }^{\mathrm{a}}$ And Noureddine OuelaA \\ Faculté des Sciences et de la Technologie, Université 8 mai 1945 Guelma, Algérie
}

Received 28 February 2016, Accepted 2 October 2016

\begin{abstract}
Nowadays, rotating machines tend to operate at high speeds and sometimes, in complex situations such as on moving support. Thus, accurate prediction of their dynamic behavior must be made to ensure a reliable design. In this paper a finite element model is developed, to simulate the dynamic behavior of a flexible rotor with constant/variable spin speed, supported by a rigid base undergoing a general movement. Simulation results are presented and discussed for different cases of base movements and running speed variations.
\end{abstract}

Key words: Rotordynamics / base motion / variable spin speed / finite element method / simulation

\section{Introduction}

Rotor-bearing systems are parts of industrial machines such as turbines, compressors, pumps and engines. Excessive vibrations of rotating machines can seriously affect their integrity. Hence, the importance of studying the behavior of these systems under various conditions.

A wide variety of studies concerning the dynamic behavior of rotors on a fixed base are encountered in literature [1-3]. However, works interested to the movement of the base are relatively rare. F.Lin and G.Meng [4] used a Jeffcott model to study the dynamic behavior of a rotor in a maneuvering aircraft, where the rotor is assumed running at a constant angular speed or a constant acceleration while the aircraft maneuvers in a vertical plane with equal pitching angle and flight path inclination. Duchemin [5] used a finite element model to study the dynamic behavior of rotors in bending, running at constant speed, excited by their base, in parallel to an experimental study that aims to validate the model. Different calculation-measurement comparisons are made. Duchemin et al. [6] investigated the dynamic behavior of a rotor subject to base excitation. A mathematical model is developed where equations are obtained using Lagrange method with Rayleigh-Ritz approximation. The method of multiple scales is then applied to study the instability zones when the base is subjected to a sinusoidal rotation. Experimental results are also presented to validate the analytical and numerical results. Driot et al. [7] used the model presented in Reference [5] to develop a theo-

\footnotetext{
${ }^{a}$ Corresponding author: ryadbouziani@yahoo.fr
}

retical study based on both the multiple scales method and the normal form approach. An experimental setup was developed. The results obtained from experimental and numerical studies are quite satisfactory. Das et al. [8] investigated the active vibration control with electromagnetic exciters of an unbalanced disk- shaft system on a moving base. Lee et al. [9] proposed a transient response analysis technique, applying the generalized finite element modeling method of a rotor-bearing system considering a base-transferred shock force along with the state space Newmark scheme. El-Saeidy et al. [10] presented a formulation for the dynamic analysis of rigid rotors with elastic linear/nonlinear bearings subject to unbalance and harmonic base excitations along or around lateral directions. Analytical and numerical integrations of the equations of motion are performed and results are discussed. Dakel et al. [11] used a finite element model based on the Timoshenko beam theory to analyze the steady-state dynamic behavior of on-board rotors mounted on rigid bearings. The model takes into account possible geometric asymmetry of the disk and/or the shaft. Numerical examples of symmetric and asymmetric rotors, whose rigid base is subjected to combined constant angular velocity and harmonic translation, are studied. Dakel et al. [12] studied, using the finite element method, the dynamic behavior of an on-board rotor mounted on hydrodynamic journal bearings in presence of rigid support movements. Two models of the hydrodynamic bearings are considered: linear and nonlinear. The influence of sinusoidal motions of the support on the dynamics of the system is analyzed by means of stability charts, orbits of the rotor, time history responses, fast Fourier transforms, bifurcation 


\section{Nomenclature}

\begin{tabular}{|lll|}
\hline$m_{d}$ & Disk mass & $\mathrm{Kg}$ \\
$m_{u} d$ & Mass unbalance & $\mathrm{Kg}-\mathrm{m}$ \\
$I_{d}, I_{p}$ & Transverse and polar mass inertia of the disk & $\mathrm{Kg} \cdot \mathrm{m}^{-2}$ \\
$I$ & Transverse area moment of inertia of a circular section shaft & $\mathrm{m}^{4}$ \\
$R_{0}\left(x_{0}, y_{0}, z_{0}\right)$ & Inertial frame & \\
$R_{b}\left(x_{b}, y_{b}, z_{b}\right)$ & Frame fixed to the moving base & \\
$R(x, y, z)$ & Frame fixed to the disk & $\mathrm{N} . \mathrm{m}$ \\
$T_{d}, T_{s}, T_{u}$ & Kinetic energy of disk, shaft and mass unbalance & $\mathrm{m}$ \\
$u, w$ & Components of displacement of a point in the shaft w.r.t. $R_{b}$ & $\mathrm{~m} . \mathrm{s}^{-1}$ \\
$\dot{u}_{a}, \dot{v}_{a}, \dot{w}_{a}$ & Components of absolute velocity of a point in the centerline of the shaft expressed in $R_{b}$ & $\mathrm{~m} . \mathrm{s}^{-1}$ \\
$\dot{u}_{u}, \dot{v}_{u}, \dot{w}_{u}$ & Components of velocity of mass unbalance expressed in $R_{b}$ & $\mathrm{~N} . \mathrm{m}$ \\
$U_{s}$ & Strain energy of the shaft & \\
$\lambda_{1}, \lambda_{2}, \lambda_{3}, \lambda_{4}$ & Parameters associated with the frame $R_{b}$ motion & $\mathrm{rd} . \mathrm{s}^{-1}$ \\
$\dot{\Gamma}_{x}, \dot{\Gamma}_{y}, \dot{\Gamma}_{z}$ & Components of angular velocity of the frame $R_{b}$ w.r.t $R_{0}$ expressed in $R_{b}$ & $\mathrm{rd}$ \\
$\phi, \psi$ & Euler angles between $R$ and $R_{b}$ frames & $\mathrm{kg}$ \\
{$\left[\mathbf{M}_{\mathbf{d}}\right],\left[\mathbf{M}_{s}\right]$} & Mass matrices of disk and shaft elements & $\mathrm{N} . \mathrm{s} . \mathrm{m}^{-1}$ \\
{$\left[\mathbf{C}_{d}\right],\left[\mathbf{C}_{s}\right]$} & Gyroscopic matrices of on-board disk and shaft elements & $\mathrm{N} . \mathrm{m}^{-1}$ \\
{$\left[\mathbf{K}_{d}\right]\left[\mathbf{K}_{s}\right]$} & Stiffness matrices of on-board disk and shaft elements & $\mathrm{m}$ \\
$\{\delta\}$ & Nodal displacement vector & $\mathrm{m}$ \\
$\{\mathbf{q}\}$ & Global displacement vector & $\mathrm{N}$ \\
$\left\{\mathbf{F}_{d}\right\},\left\{\mathbf{F}_{s}\right\}$ & Force vectors of on-board disk and shaft elements & \\
\hline
\end{tabular}

diagrams and Poincaré maps. Han and Chu [13] studied the dynamic behavior of cracked rotor-bearing system subjected to harmonic base angular motions using the harmonic balance method. The response spectra, orbits of shaft center and frequency response for the base excited rotor-bearing system with open and breathing cracks were discussed. Han and Chu [14] studied the dynamic behaviors of a geared rotor system under timeperiodic base motions. The effects of various base angular motions on both frequency response and response spectra were discussed. Han and Chu [15] analyzed the parametric instability of flexible rotor-bearing system under timeperiodic base angular motions. Instability computations for a rotor-bearing system with base motions are conducted. The effects of rotating speed, amplitudes of base motion and phases between two base motions on both the primary and combination instability regions were discussed.

Previous works of other authors [5, 6, 11-15] investigated the steady-state dynamic behavior of on-board rotors subject to base motion. In this paper, we use similar representations of rotor and base movements to study onboard rotors running at variable rotational speed. For this aim, a finite element model of a geometrically symmetric rotor is developed, and a computer code is elaborated, on the basis of a software accompanying [3]. This allows analyzing the dynamic behavior of on-board rotors under variable rotational speed such as during startup and shut down.

\section{Finite element model}

To describe the movement of the rotor, with respect to the moving rigid base, three reference frames are considered (Fig. 1).

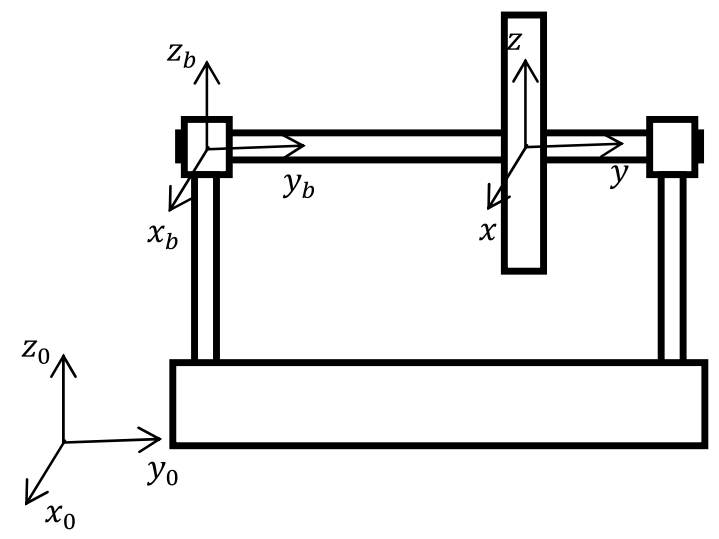

Fig. 1. Rotor-bearing system on moving base with different coordinate systems.

$R_{0}\left(x_{0}, y_{0}, z_{0}\right):$ Inertial frame.

$R_{b}\left(x_{b}, y_{b}, z_{b}\right)$ : Frame fixed to the rigid moving base.

$R(x, y, z)$ : Frame fixed to the disk.

\subsection{Linear and angular velocities}

The expressions of the components of velocity vectors to be used in energies expressions are presented here after $[5,6,11]$ :

- The components of the absolute velocity vector of a point in the centerline of the shaft expressed in $R_{b}$ are:

$$
\begin{aligned}
\dot{u}_{a} & =\dot{x}_{b}+\dot{u}+\dot{\Gamma}_{y}\left(z_{b}+w\right)-\dot{\Gamma}_{z}\left(y_{b}+y\right) \\
\dot{v}_{a} & =\dot{y}_{b}+\dot{\Gamma}_{z}\left(x_{b}+u\right)-\dot{\Gamma}_{x}\left(z_{b}+w\right) \\
\dot{w}_{a} & =\dot{z}_{b}+\dot{w}+\dot{\Gamma}_{x}\left(y_{b}+y\right)-\dot{\Gamma}_{y}\left(x_{b}+u\right)
\end{aligned}
$$


where $u, w$ are the components of displacement of a point in the centerline of the shaft w.r.t. $R_{b}, \dot{\Gamma}_{x}, \dot{\Gamma}_{y}, \dot{\Gamma}_{z}$ are the components of angular velocity of the fram $R_{b}$ w.r.t $R_{0}$ expressed in $R_{b}$.

- The components of the angular velocity vector of the frame $R$ w.r.t $R_{0}$ written in $R$ are

$$
\begin{aligned}
\omega_{x} & =\left(\dot{\theta}+\dot{\Gamma}_{x} \cos \psi+\dot{\Gamma}_{y} \sin \psi\right) \cos \phi \\
& -\left(\dot{\Gamma}_{x} \sin \psi-\dot{\Gamma}_{y} \cos \psi\right) \sin \theta \sin \phi \\
& -\left(\dot{\Gamma}_{z}+\dot{\psi}\right) \cos \theta \sin \phi \\
\omega_{y} & =-\left(\dot{\Gamma}_{x} \sin \psi-\dot{\Gamma}_{y} \cos \psi\right) \cos \theta+\left(\dot{\Gamma}_{z}+\dot{\psi}\right) \sin \theta+\dot{\phi} \\
\omega_{z} & =\left(\dot{\theta}+\dot{\Gamma}_{x} \cos \psi+\dot{\Gamma}_{y} \sin \psi\right) \sin \phi \\
& +\left(\dot{\Gamma}_{x} \sin \psi-\dot{\Gamma}_{y} \cos \psi\right) \sin \theta \cos \phi \\
& +\left(\dot{\Gamma}_{z}+\dot{\psi}\right) \cos \theta \cos \phi
\end{aligned}
$$

where $\phi, \theta, \psi$ are Euler angles between $R$ and $R_{b}$ frames.

- The components of velocity of mass unbalance expressed in $R_{b}$ are:

$$
\begin{aligned}
\dot{u}_{u} & =\dot{x}_{b}+\dot{u}+\dot{\Gamma}_{y}\left(z_{b}+w+d \cos (\phi+\alpha)\right) \\
& -\dot{\Gamma}_{z}\left(y_{b}+y_{d}\right)+d \dot{\phi} \cos (\phi+\alpha) \\
\dot{v}_{u} & =\dot{y}_{b}+\dot{\Gamma}_{z}\left(x_{b}+u+d \sin (\phi+\alpha)\right) \\
& -\dot{\Gamma}_{x}\left(z_{b}+w+d \cos (\phi+\alpha)\right) \\
\dot{w}_{u} & =\dot{z}_{b}+\dot{w}-\dot{\Gamma}_{y}\left(x_{b}+u+d \sin (\phi+\alpha)\right) \\
& +\dot{\Gamma}_{x}\left(y_{b}+y_{d}\right)-d \dot{\phi} \sin (\phi+\alpha)
\end{aligned}
$$

where: $y_{d}$ denotes the disk position in $\mathrm{y}$ axis and $\alpha$ the initial phase angle of the unbalance; and $d$ is the distance of unbalance from the geometric center of the shaft.

\subsection{Energies}

Applying Lagrange method to obtain the equations of motion of the rotor-bearing system, requires the determination of kinetic and strain energies of each element.

- The kinetic energy of the rigid disk is

$$
\begin{aligned}
T_{d} & =\left.\frac{1}{2} m_{d}\left(\dot{u}_{a}^{2}+\dot{v}_{a}^{2}+\dot{w}_{a}^{2}\right)\right|_{y=y_{d}} \\
& +\frac{1}{2}\left(\mathrm{I}_{d} \omega_{x}^{2}+\mathrm{I}_{p} \omega_{y}^{2}+I_{d} \omega_{z}^{2}\right)
\end{aligned}
$$

where $m_{d}$ is the disk mass and $I_{d}$ and $I_{p}$ are transverse and polar mass inertia of the disk.

- The kinetic energy of the symmetric shaft element is written a

$$
\begin{aligned}
T_{s} & =\frac{1}{2} \int_{0}^{L} \rho s\left(\dot{u}_{a}^{2}+\dot{v}_{a}^{2}+\dot{w}_{a}^{2}\right) \mathrm{d} y \\
& +\frac{1}{2} \int_{0}^{L} \rho I\left(\omega_{x}^{2}+2 \omega_{y}^{2}+\omega_{z}^{2}\right) \mathrm{d} y
\end{aligned}
$$

where $I$ is the transverse area inertia of the shaft.
- The strain energy of the symmetric shaft can be written as:

$$
U_{s}=\frac{E I}{2} \int_{0}^{L}\left[\left(\frac{\partial^{2} u}{\partial y^{2}}\right)^{2}+\left(\frac{\partial^{2} w}{\partial y^{2}}\right)^{2}\right] \mathrm{d} y
$$

- The kinetic energy of the ass unbalance is

$$
T_{u}=\frac{1}{2} m_{u}\left(\dot{u}_{u}^{2}+\dot{v}_{u}^{2}+\dot{w}_{u}^{2}\right)
$$

where $m_{u}$ is the mass unbalance of the rotor.

\subsection{Finite elements for the rotor components}

\subsubsection{Disk}

At a given node the rotor has four degrees of freedom (d.o.f) : $u, w, \theta$ and $\psi$. Then, if the nodal displacement vector of the disk center is

$$
\{\delta\}=[u, w, \theta, \psi]^{t}
$$

Applying Lagrange equations yields:

$$
\begin{aligned}
& \frac{d}{d t}\left(\frac{\partial T_{d}}{\partial\{\dot{\delta}\}}\right)-\frac{\partial T_{d}}{\partial\{\delta\}} \\
& \quad=\left[\mathbf{M}_{d}\right]\{\ddot{\delta}\}+\left[\mathbf{C}_{d}\right]\{\dot{\delta}\}+\left[\mathbf{K}_{d}\right]\{\delta\}-\left\{\mathbf{F}_{d}\right\}
\end{aligned}
$$

where

$$
\begin{gathered}
{\left[\mathbf{C}_{d}\right]=\dot{\phi}\left[\mathbf{C}_{d_{1}}\right]+\dot{\Gamma}_{y}\left[\mathbf{C}_{d_{2}}\right]} \\
{\left[\mathbf{K}_{d}\right]=\left[\begin{array}{cc}
{\left[\mathbf{K}_{d 11}\right]} & {[0]} \\
{[0]} & {\left[\mathbf{K}_{d 22}\right]}
\end{array}\right]}
\end{gathered}
$$

with

$$
\begin{gathered}
{\left[\mathbf{K}_{d 11}\right]=m_{d}\left[\begin{array}{cc}
-\left(\dot{\Gamma}_{y}^{2}+\dot{\Gamma}_{z}^{2}\right) & \dot{\Gamma}_{x} \dot{\Gamma}_{z}+\ddot{\Gamma}_{y} \\
\dot{\Gamma}_{x} \dot{\Gamma}_{z}-\ddot{\Gamma}_{y}-\left(\dot{\Gamma}_{x}^{2}+\dot{\Gamma}_{y}^{2}\right)
\end{array}\right]} \\
{\left[\mathbf{K}_{d 22}\right]=\left(I_{d}-I_{p}\right)\left[\begin{array}{cc}
\dot{\Gamma}_{z}^{2}-\dot{\Gamma}_{y}^{2} & -\dot{\Gamma}_{x} \dot{\Gamma}_{z}+\ddot{\Gamma}_{y} \\
-\dot{\Gamma}_{x} \dot{\Gamma}_{z}-\ddot{\Gamma}_{y} & \dot{\Gamma}_{x}^{2}-\dot{\Gamma}_{y}^{2}
\end{array}\right]} \\
\left.+I_{p}\left[\begin{array}{cc}
\dot{\phi} \dot{\Gamma}_{y} & \ddot{\Gamma}_{y} \\
\ddot{\phi} & \dot{\phi} \dot{\Gamma}_{y}
\end{array}\right] \begin{array}{c}
m_{d}\left(\lambda_{1}+\lambda_{3} y_{d}\right) \\
m_{d}\left(\lambda_{2}+\lambda_{4} y_{d}\right) \\
-I_{p} \dot{\Gamma}_{z}\left(\dot{\Gamma}_{y}+\dot{\phi}\right)+I_{d} \lambda_{4} \\
I_{p} \dot{\Gamma}_{x}\left(\dot{\Gamma}_{y}+\dot{\phi}\right)-I_{d} \lambda_{3}
\end{array}\right\} \\
\left\{\mathbf{F}_{d}\right\}=-
\end{gathered}
$$




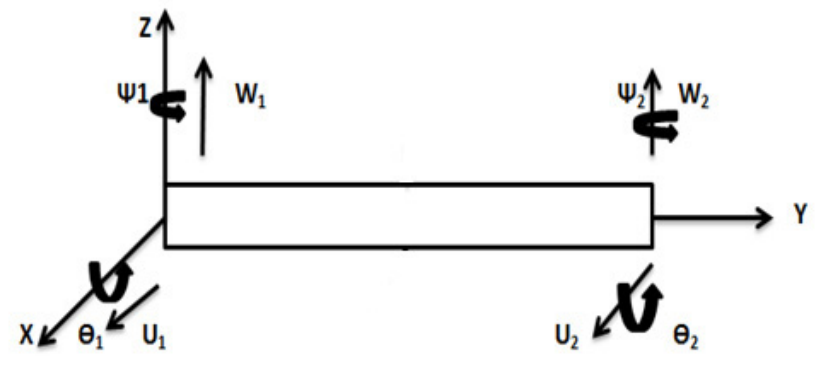

Fig. 2. Four d.o.f beam element.

The matrices and parameters used in these equations and not expressed above are given in Appendix.

\subsubsection{Shaft}

The shaft with a constant circular cross-section is modeled using two-noded Euler-Bernoulli beam finite elements (Fig. 2).

The nodal displacement vector of the element is

$$
\{\delta\}=\left[u_{1}, w_{1}, \theta_{1}, \psi_{1}, u_{2}, w_{2}, \theta_{2}, \psi_{2}\right]^{t}
$$

Applying Lagrange equations gives:

$$
\begin{aligned}
& \frac{d}{d t}\left(\frac{\partial T_{s}}{\partial\{\dot{\delta}\}}\right)-\frac{\partial T_{s}}{\partial\{\delta\}} \\
& \quad=\left[\mathbf{M}_{s}\right]\{\ddot{\delta}\}+\left[\mathbf{C}_{s}\right]\{\dot{\delta}\}+\left[\mathbf{K}_{s}\right]\{\delta\}-\left\{\mathbf{F}_{s}\right\}
\end{aligned}
$$

where

$$
\begin{aligned}
{\left[\mathbf{M}_{s}\right] } & =\left[\mathbf{M}_{1}\right]+\left[\mathbf{M}_{s 1}\right] \\
{\left[\mathbf{C}_{s}\right] } & =2 \dot{\phi}\left[\mathbf{C}_{1}\right]+2 \dot{\Gamma}_{y}\left[\mathbf{C}_{2}\right] \\
{\left[\mathbf{K}_{s}\right] } & =\left[\mathbf{K}_{1}\right]-\left[\mathbf{K}_{3}\right]+\ddot{\Gamma}_{y}\left[\mathbf{C}_{2}\right]+\dot{\Gamma}_{x} \dot{\Gamma}_{z}\left[\mathbf{K}_{2}\right] \\
& +2 \dot{\Gamma}_{y}\left(\dot{\phi}+\dot{\Gamma}_{y}\right)\left[\mathbf{M}_{s 1}\right]-\left(\ddot{\Gamma}_{y}+\dot{\Gamma}_{x} \dot{\Gamma}_{z}\right)\left[\mathbf{K}_{4}\right]+\ddot{\phi}\left[\mathbf{K}_{5}\right]
\end{aligned}
$$

Deriving the strain energy w.r.t. displacements gives:

$$
\frac{\partial U_{s}}{\partial\{\delta\}}=\left[\mathbf{K}_{s 1}\right]\{\delta\}
$$

where the matrices used in these expressions are given in Appendix.

\subsection{Mass unbalance}

Applying Lagrange equations to kinetic energy of mass unbalance, and neglecting $m_{u}$ w.r.t $m_{d}$ yields:

$$
\begin{aligned}
& \frac{\mathrm{d}}{\mathrm{d} t}\left(\frac{\partial T_{u}}{\partial\{\dot{\delta}\}}\right)-\frac{\partial T_{u}}{\partial\{\delta\}} \\
& =m_{u} d\left\{\begin{array}{c}
\ddot{\phi}+\ddot{\Gamma}_{y}+\dot{\Gamma}_{x} \dot{\Gamma}_{z} \\
-\left(\dot{\phi}+\dot{\Gamma}_{y}\right)^{2}-\dot{\Gamma}_{x}^{2} \\
0 \\
0
\end{array}\right\} \cos (\phi+\alpha) \\
& +m_{u} d\left\{\begin{array}{c}
-\left(\dot{\phi}+\dot{\Gamma}_{y}\right)^{2}-\dot{\Gamma}_{z}^{2} \\
-\dot{\phi}-\ddot{\Gamma}_{y}+\dot{\Gamma}_{x} \dot{\Gamma}_{z} \\
0 \\
0
\end{array}\right\} \sin (\phi+\alpha)
\end{aligned}
$$

\subsubsection{Bearings}

The forces at the bearings are given by the expression

$$
\begin{aligned}
{\left[\begin{array}{c}
F_{u} \\
F_{\psi} \\
F_{w} \\
F_{\theta}
\end{array}\right]=} & -\left[\begin{array}{cccc}
k_{x x} & 0 & k_{x z} & 0 \\
0 & 0 & 0 & 0 \\
k_{z x} & 0 & k_{z z} & 0 \\
0 & 0 & 0 & 0
\end{array}\right]\left[\begin{array}{c}
u \\
\theta \\
w \\
\psi
\end{array}\right] \\
& -\left[\begin{array}{cccc}
c_{x x} & 0 & c_{x z} & 0 \\
0 & 0 & 0 & 0 \\
c_{z x} & 0 & c_{z z} & 0 \\
0 & 0 & 0 & 0
\end{array}\right]\left[\begin{array}{c}
\dot{u} \\
\dot{\theta} \\
\dot{w} \\
\dot{\psi}
\end{array}\right]
\end{aligned}
$$

The first matrix is a stiffness matrix and the second a viscous damping matrix. These matrices are generally asymmetric and the terms of the matrices can vary significantly as a function of the speed of rotation [1].

\subsubsection{Equations of motion and solution}

After assembling the equations of shaft elements, rigid disks and mass unbalance, taking into account the characteristics of bearings, the equation of motion for the complete rotor system is defined as follows:

$$
[\mathbf{M}]\{\ddot{q}(t)\}+[\mathbf{C}]\{\dot{q}(t)\}+[\mathbf{K}]\{\mathbf{q}(t)\}=\{\mathbf{F}(t)\}
$$

where $\{\mathbf{q}(t)\}$ is a vector containing all the nodal displacements, $[\mathbf{M}]$ is the mass matrix of the rotor, $[\mathbf{C}]$ is the asymmetric matrix including gyroscopic and base motion effects and bearing damping, $[\mathbf{K}]$ the stiffness matrix of the rotor including bending stiffness, base motion effect, rotational speed variations effects and bearing stiffness. Finally, $\{(\mathbf{F} t)\}$ is the global external force vector, depending on rotational speed and acceleration, containing the effects of mass unbalance and the inertia force due to the base motion .

It is worth noting that the difference between the equations of motion obtained in this work and those in 


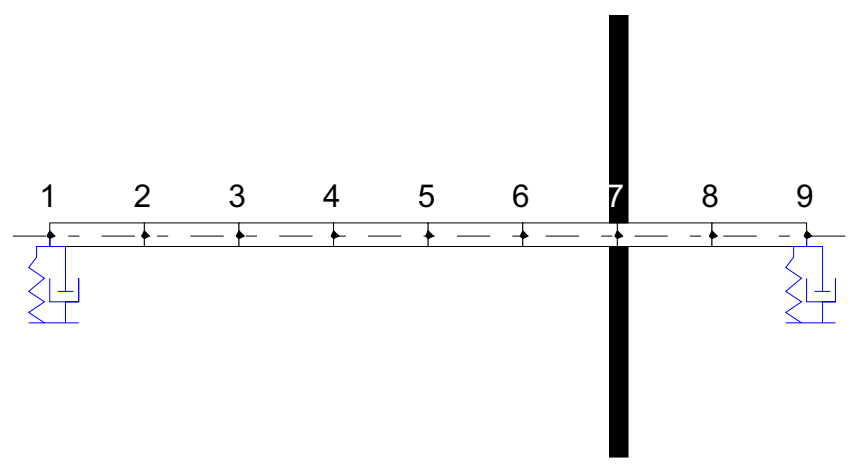

Fig. 3. Finite element discretization for the first configuration of the rotor (Tab. 1).

the works of other authors dealing with on-board rotors $[5,11,12]$, is that the spin speed here is variable and then terms containing rotating acceleration, coming from Equations (13), (19) and (21), are present.

The solutions of the set of differential Equations (23) are obtained using the Newmark method based on the average constant acceleration scheme.

\section{Results and discussions}

\subsection{Validation for constant rotational speed}

To validate the developed code, several tests have been performed on two configurations of a rotor-bearing system. In each case, the system consists of a single disk placed on a flexible shaft which is supported on two bearings at its ends.

The overall rotor-bearing system is placed on a base that may be fixed or in movement. In the case of rotational movements the coordinates of the rotation center of the base are considered null.

The geometry and the material properties of the rotor-bearing system used in the two configurations are given in Tables 1 and 2 .

\subsubsection{First configuration of the rotor (Tab. 1)}

For the sake of comparison, the first configuration of the rotor is taken from $[6,7]$ as shown in Table 1.

The shaft is discretized into eight equal length 2-noded beam finite elements. The disk, with mass unbalance, is located at node 7 and the bearings with null cross coefficients are at nodes 1 and 9 as shown in Figure 3 .

In Figure 4, are presented the orbits, obtained from simulations using the program developed in this work, for a constant spin speed of the rotor $\Omega=20 \mathrm{~Hz}(1200 \mathrm{rpm})$ and a sinusoidal rotation of the base about $x$-axis of amplitude $2 \times 10^{-5} \mathrm{rd}$, with two frequencies: $60 \mathrm{~Hz}$ and $80 \mathrm{~Hz}$ and the results are identical to [7].

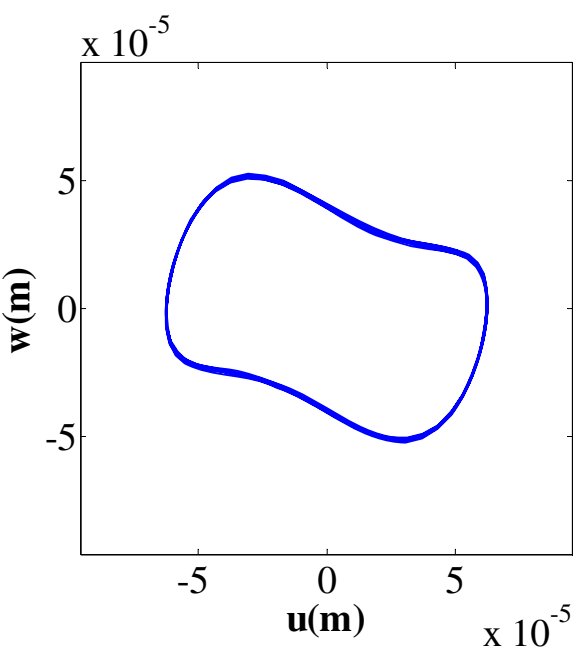

(a)

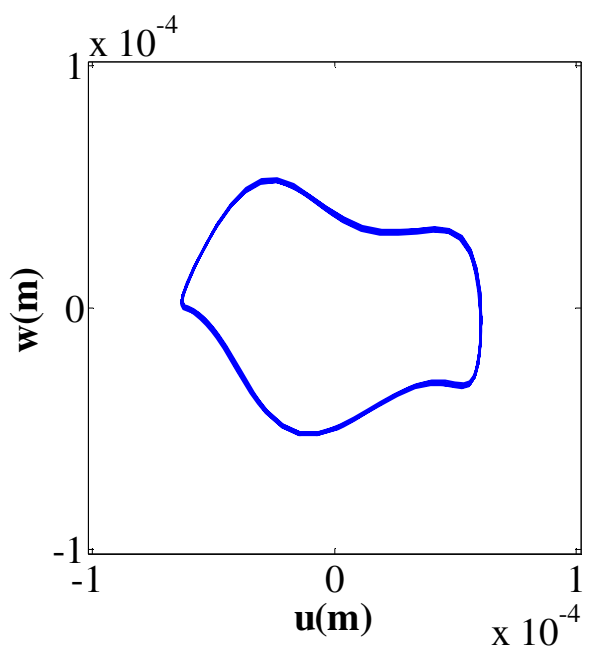

(b)

Fig. 4. Orbits at the middle of the shaft of a rotor running at $\Omega=20 \mathrm{~Hz}(1200 \mathrm{rpm})$ subject to sinusoidal rotation of the base about $x$-axis of amplitude $2 \times 10^{-5}$ rd and a frequency (a) $60 \mathrm{~Hz}($ b) $80 \mathrm{~Hz}$.

\subsubsection{Second configuration of the rotor (Tab. 2)}

In the second test, the configuration of the rotor in Reference [11] is used as shown in Table 2. The shaft is discretized into twelve equal length 2 -noded beam finite elements. The disk, with mass unbalance, is located at node 5 and the two short rigid bearings at nodes 1 and 13 as shown in Figure 5.

This second configuration of the rotor-bearing system has been tested for three combinations of base movements [11] with a rotation speed of $1500 \mathrm{rpm}(25 \mathrm{~Hz})$.

In the three cases, the base movement consists of a vertical sinusoidal translation of magnitude $10^{-6} \mathrm{~m}$ combined to a rotation of a frequency of $5 \mathrm{~Hz}$ about $y$-axis.

The frequencies of the translation motion are $100 \mathrm{~Hz}$, $120 \mathrm{~Hz}$ and $200 \mathrm{~Hz}$, respectively. As shown in Figure 6, the orbits obtained are identical to those in Reference [11]. 
Table 1. Simulation parameters for the first configuration of the rotor.

\begin{tabular}{ccc}
\hline Symbol & Quantity & Value \\
\hline$L$ & Shaft length & $0.4 \mathrm{~m}$ \\
$R_{1}$ & Shaft radius & $0.005 \mathrm{~m}$ \\
$R_{2}$ & Disk radius & $0.1 \mathrm{~m}$ \\
$h$ & Disk thickness & $0.01 \mathrm{~m}$ \\
$E$ & Young modulus & $2.05 \times 10^{11} \mathrm{~N} . \mathrm{m}^{-2}$ \\
$\rho$ & Mass per unit volume & $7800 \mathrm{~kg} \cdot \mathrm{m}^{-3}$ \\
$m_{u} d$ & Residual unbalance & $2.5 \times 10^{-4} \mathrm{~kg} \mathrm{~m}$ \\
$k_{x x}$ & Bearing stiffness along $x$-axis & $2.1 \times 10^{5} \mathrm{~N} \cdot \mathrm{m}^{-1}$ \\
$k_{z z}$ & Bearing stiffness along $z$-axis & $1.2 \times 10^{6} \mathrm{~N} \cdot \mathrm{m}^{-1}$ \\
$c_{x x}=c_{z z}$ & Bearing damping along $x$ and $z$ axis & $15000 / \Omega \mathrm{N} \cdot \mathrm{m}^{-1} \cdot \mathrm{s}^{-1}$ \\
\hline
\end{tabular}

Table 2. Simulation parameters for the second configuration of the rotor.

\begin{tabular}{ccc}
\hline Symbol & Quantity & Value \\
\hline$L$ & Shaft length & $0.4 \mathrm{~m}$ \\
$R_{1}$ & Shaft radius & $0.01 \mathrm{~m}$ \\
$R_{2}$ & Disk radius & $0.15 \mathrm{~m}$ \\
$h$ & Disk thickness & $0.03 \mathrm{~m}$ \\
$E$ & Young modulus & $2 \times 10^{11}{\mathrm{~N} . \mathrm{m}^{-2}}^{-3}$ \\
$\rho$ & Mass per unit volume & $7800 \mathrm{~kg} \cdot \mathrm{m}^{-3}$ \\
$m_{u} d$ & Residual unbalance & $1.5 \times 10^{-4} \mathrm{~kg} \mathrm{~m}$ \\
\hline
\end{tabular}

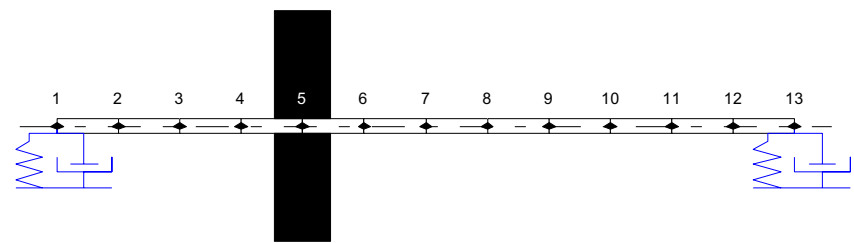

Fig. 5. Finite element discretization for the second configuration of the rotor (Tab. 2).

\subsection{Application to variable rotational speed}

After validating the model for constant spin speed, the case of variable spin speed is dealt with. Two laws of variation are considered: linear and exponential. For linear variation, the configuration of the rotor described in table 1 is used, whereas for the exponential case the configuration of the rotor described in Table 2 is considered.

Campbell diagrams for the two configurations of the rotor are plotted in Figures 7 and 8 .

\subsubsection{Linear law of spin speed}

The case of a fixed base is first considered and then different cases of base movements are dealt with.

Test 1: The rotational speed of the rotor is variable with constant acceleration (deceleration), and the base is fixed.

In Figure 9 is presented the response at the middle of the shaft of the rotor described in Table 1 mounted on a fixed base, running with a variable rotational speed, during $5 \mathrm{~s}$, with constant acceleration (100 rd.s $\left.{ }^{-2}\right)$ (Fig. 9a), and with constant deceleration $\left(-100{\mathrm{rd} . \mathrm{s}^{-2}}^{2}\right.$ (Fig. 9b); and in Figure 10 are superimposed the two response graphs corresponding to acceleration and deceleration as function of rotational speed. Figure 10 shows clearly that the maximum response does not occur at the same rotational speed in acceleration and deceleration. Furthermore, the amplitudes are not the same. These facts are well mentioned in literature [16].

Test 2: linear spin speed, with sinusoidal rotation of the base about $x$-axis.

In Figure 11 are presented the responses at the middle of the shaft of the rotor described in Table 1 running with linear spin speed $\dot{\phi}(t)=200 t$ rd.s ${ }^{-1}$ during $5 \mathrm{~s}$ and subject to a sinusoidal rotation of the base about $x$-axis of amplitude $2 \times 10^{-4}$ rd for different frequencies: $18 \mathrm{~Hz}$, $20 \mathrm{~Hz}$ and $24 \mathrm{~Hz}$. As it can be observed, there are peaks whose positions in time axis remain unchanged (in vicinity of $1 \mathrm{~s}$ ) regardless the frequency of the base motion; they correspond to BW and FW critical speeds (natural frequencies excited by mass unbalance). In Figure 7, are indicated the values of these critical speeds $(1730 \mathrm{rpm}$ and $2030 \mathrm{rpm}$ ) on Campbell diagram. In addition to peaks corresponding to critical speeds, another peak is observed in each case; its position is changing in time axis depending on the frequency of the base motion: it is moving down when increasing the base frequency (it corresponds to a natural frequency excited by base motion). Figure 7 , representing Campbell diagram, shows the intersections between the graph of the naturel frequency, in the frequency range considered, with the horizontal lines representing base frequencies. As it can be remarked, the spin speeds values corresponding to these intersections are decreasing for increasing values of the base frequency: $20 \mathrm{~Hz}$ (7560 rpm), $22 \mathrm{~Hz}$ (6340 rpm), $24 \mathrm{~Hz}$ (5040), $26 \mathrm{~Hz}$ (3750).

Test 3: linear spin speed, with sinusoidal vertical translation of the base, for different values of base frequency.

In Figure 12, are plotted the responses of the rotor described in Table 1, running with a linear spin speed $\dot{\phi}(t)=200 t$ rd. $\mathrm{s}^{-1}$ during $5 \mathrm{~s}$ and subject to a sinusoidal vertical translation of the base of amplitude $1.5 \times 10^{-5} \mathrm{~m}$ with different frequencies: $20 \mathrm{~Hz}, 22 \mathrm{~Hz}$ and $26 \mathrm{~Hz}$. The remarks, concerning fixed and moving peaks observed for sinusoidal rotation remain valid for the case of sinusoidal translation. 
R. Bouziani and N. Ouelaa: Mechanics \& Industry 18, 308 (2017)

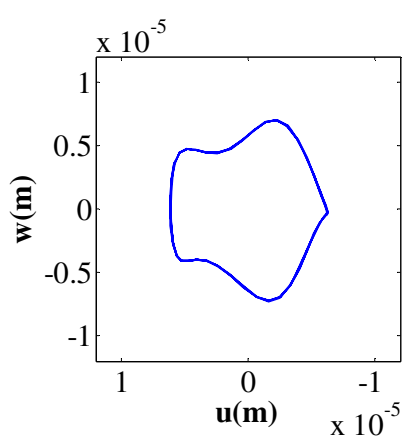

(a)

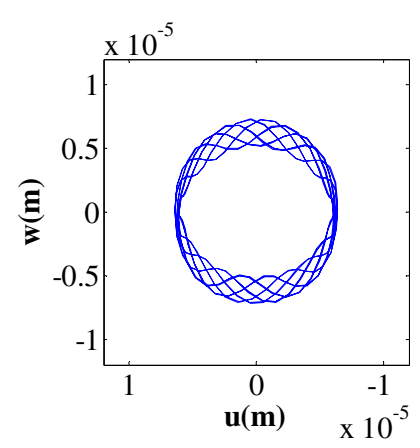

(b)

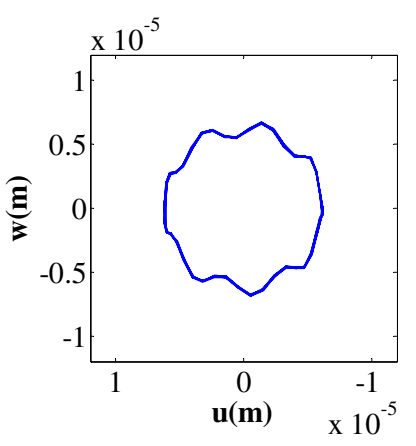

(c)

Fig. 6. Orbits at the middle of the rotor running at $1500 \mathrm{rpm}(25 \mathrm{~Hz})$ and subjected to a combined movement of the base : a vertical sinusoidal translation (amplitude $=10^{-6} \mathrm{~m}$, frequency $=$ (a) $100 \mathrm{~Hz}$, (b) $120 \mathrm{~Hz}$, (c) $200 \mathrm{~Hz}$ ), and a constant angular velocity about $y$-axis $(5 \mathrm{~Hz})$.

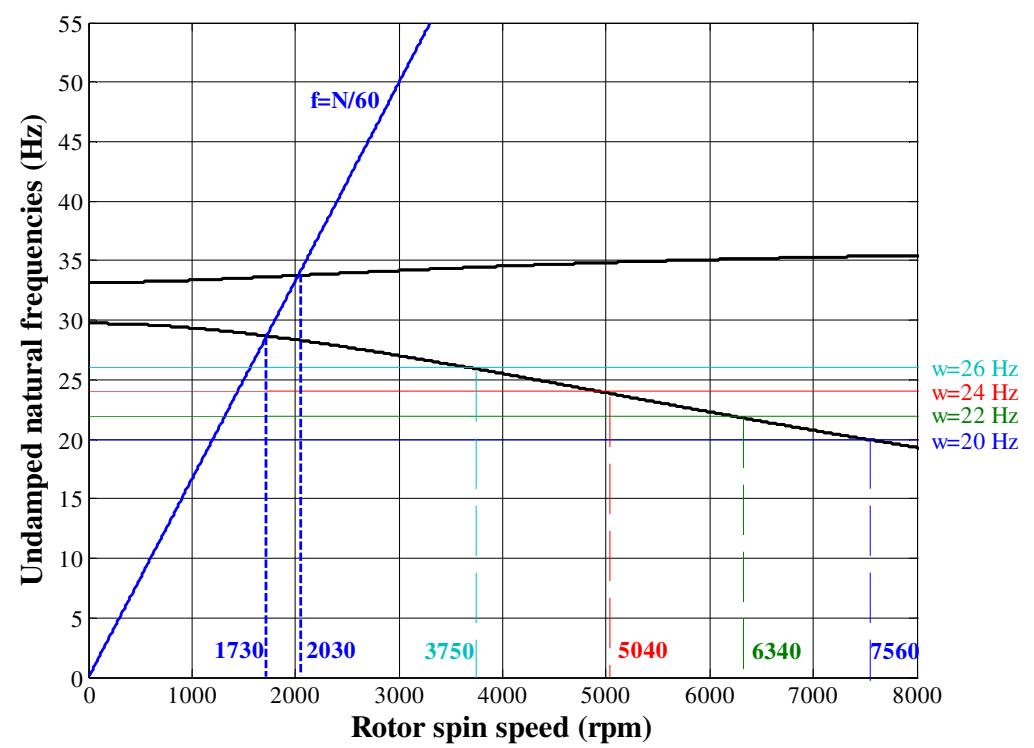

Fig. 7. Campbell diagram for the first configuration of the rotor (Tab. 1).

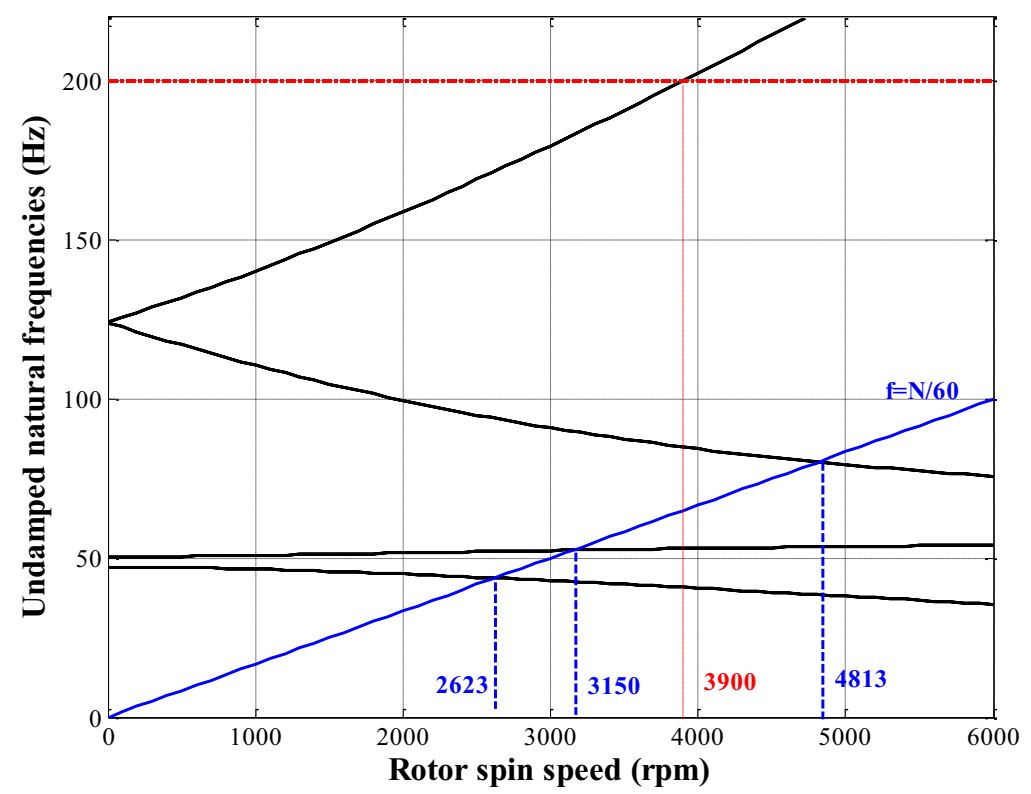

Fig. 8. Campbell diagram for the second configuration of the rotor (Tab. 2). 

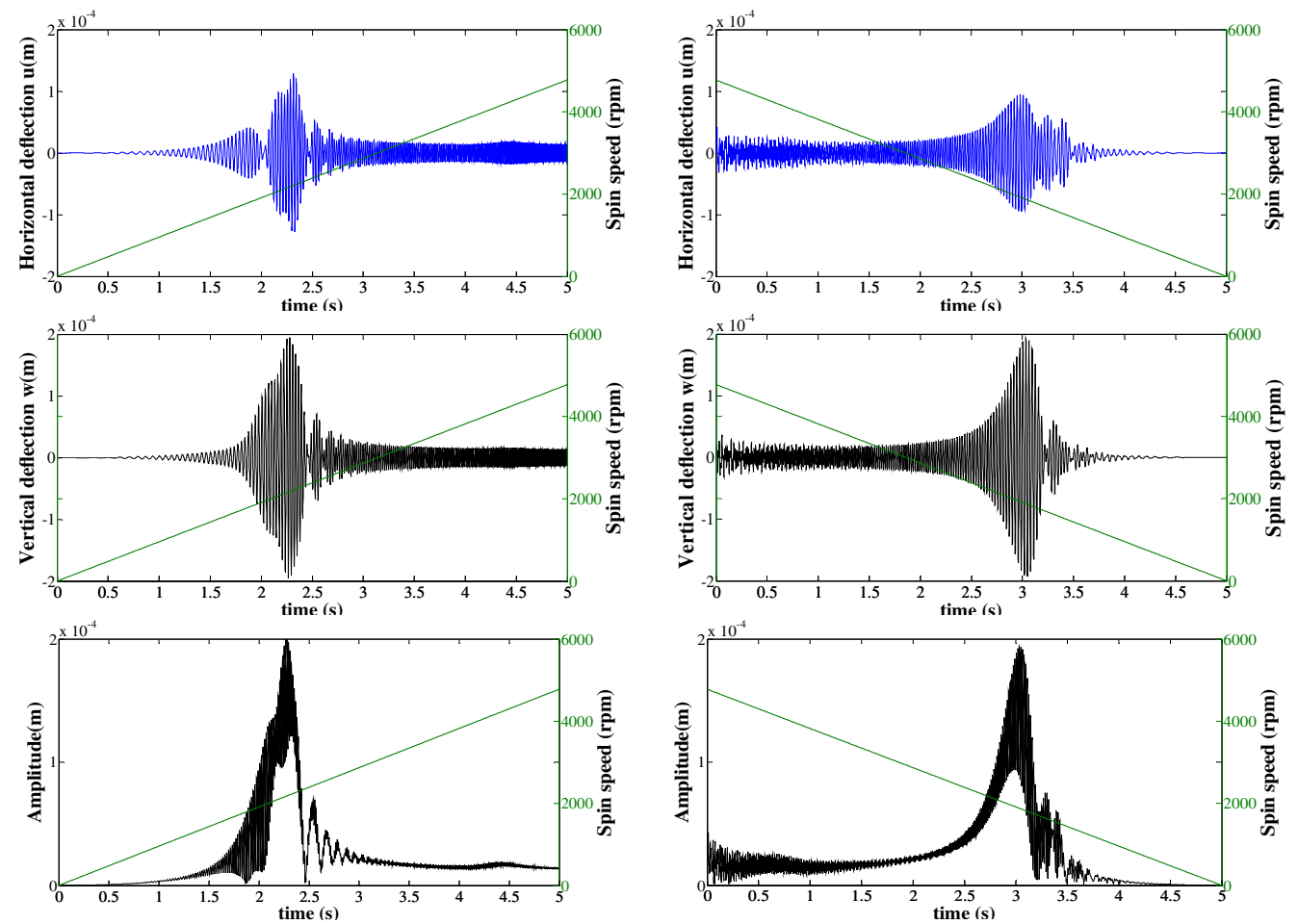

(a)

(b)

Fig. 9. Rotor mounted on a fixed base and running at variable spin speed with: (a) constant acceleration (100 rd.s $\left.{ }^{-2}\right)(\mathrm{b})$ constant deceleration $\left(-100 \mathrm{rd} \cdot \mathrm{s}^{-2}\right)$.

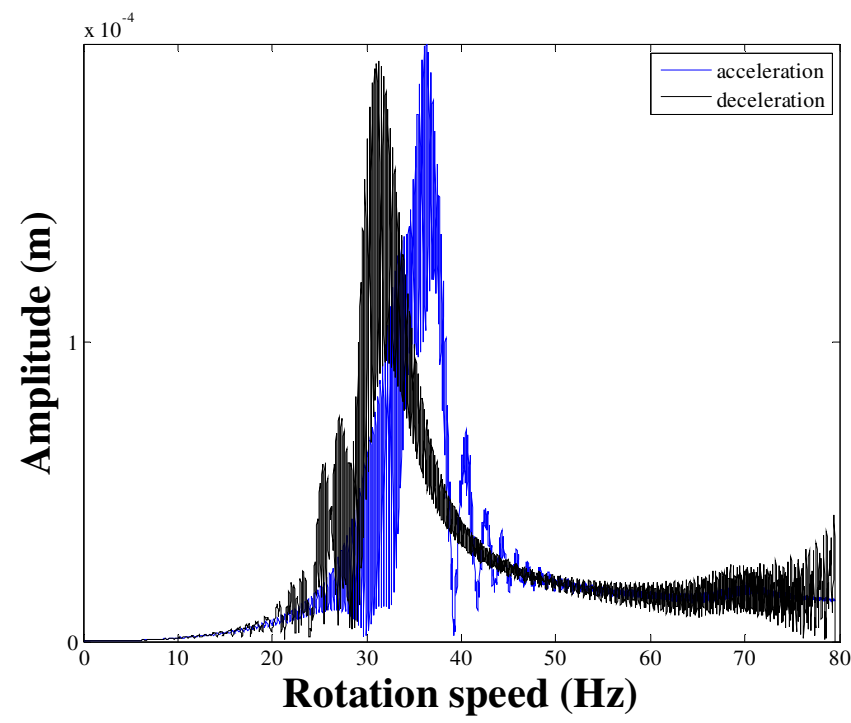

Fig. 10. Displacement amplitude in acceleration and deceleration.

In Figures 13 and 14, are presented the response along $z$-axis together with the spectrogram and the orbit for two frequencies: $20 \mathrm{~Hz}, 22 \mathrm{~Hz}$. The spectrograms show clearly that the first peak is excited by spin speed whereas the second one is due to base excitation. Concerning orbits at resonance caused by base motion, we can observe that they are changing their shapes depending on the base frequency, and that the number of vertices decreases when base frequency increases.

Test 4: linear spin speed of the rotor with vertical sinusoidal translation combined to a constant velocity rotation about $y$-axis of the base.

In test 4 , the rotor described in Table 2, with combined motions of the base: sinusoidal vertical translation of amplitude $10^{-6} \mathrm{~m}$ and a frequency $200 \mathrm{~Hz}$ with a constant velocity rotation about $y$-axis of $5 \mathrm{~Hz}$ that is studied for constant spin speed (Fig. 6a), is now subjected to a variable spin speed with constant acceleration: $500 \mathrm{rd} . \mathrm{s}^{-2}$. The displacements along $x$ nd $z$ axis are plotted in Figures $15 \mathrm{a}$ and $15 \mathrm{~b}$, and the orbit in Figure 15c.

\subsubsection{Exponential law of spin speed}

The model developed in this work can deal with any law of variation of spin speed. For a purpose of demonstration, an exponential law of spin speed is considered, the case of a fixed base is first considered and then one case of base movement is dealt with.

Test 1: Exponential spin speed of a rotor running on a fixed base.

In this case, the rotor described in Table 2 is mounted on a fixed base and is running with exponential spin speed during 5 s.

$$
\dot{\phi}(t)=534\left(1-e^{-0.983 t}\right) \operatorname{rd} . \mathrm{s}^{-1}[1]
$$


(a)

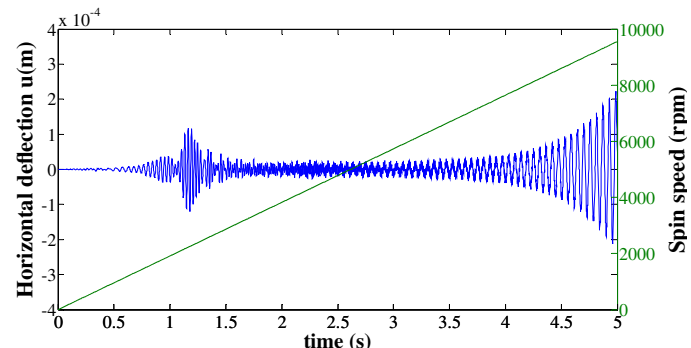

(b)

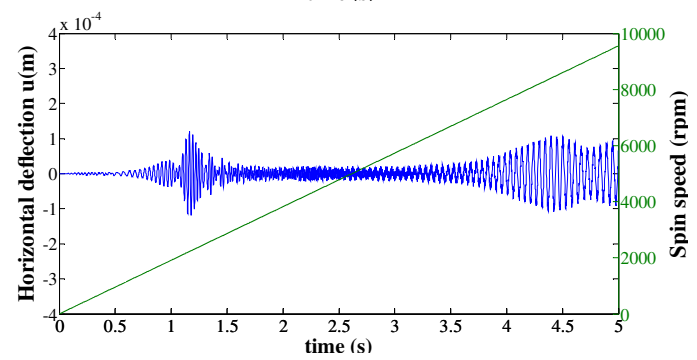

(c)

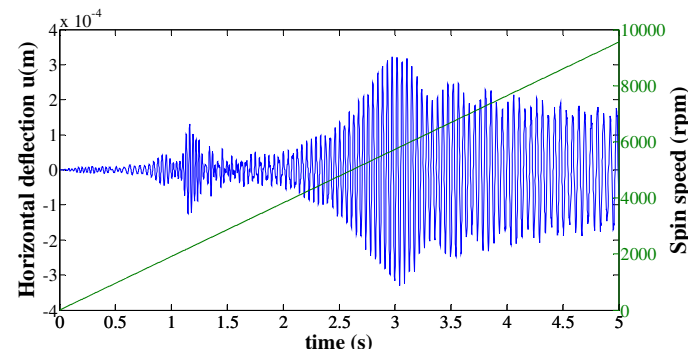

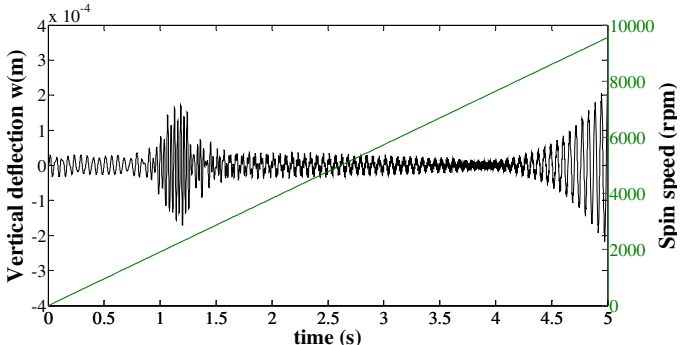
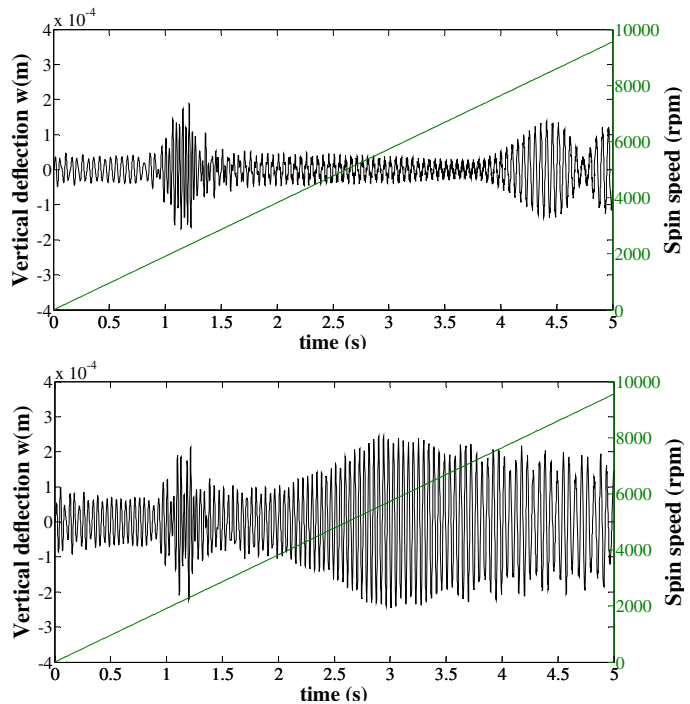

Fig. 11. Linear spin speed of a rotor $\dot{\phi}(t)=200 t$ rd.s ${ }^{-1}$ with sinusoidal rotation about $x$-axis of the base of amplitude $2 \times 10^{-4}$ rd with different frequencies: (a) $18 \mathrm{~Hz}$; (b) $20 \mathrm{~Hz}$; (c) $24 \mathrm{~Hz}$.

The horizontal and vertical deflections at the middle of the rotor and the spectrogram corresponding to vertical deflection are plotted in Figure 16.

We can observe the peaks corresponding to the first backward and forward critical speeds in $u$ and $w$ graphs and their locations in the spectrogram.

Test 2: Exponential spin speed of a rotor with vertical sinusoidal motion of the base.

The same rotor of the previous test is mounted on a moving base with a vertical translational sinusoidal motion of amplitude $10^{-5} \mathrm{~m}$ and a frequency of $200 \mathrm{~Hz}$. The running speed is exponential $\dot{\phi}(t)=$ $534\left(1-e^{-0.983 t}\right) \mathrm{rd} . \mathrm{s}^{-1}$. The horizontal and vertical deflections at the middle of the rotor and the spectrogram corresponding to vertical deflection are plotted in Figure 17.

The deflection graphs show peaks corresponding to FW and BW critical speeds occurring at the same time as those corresponding to fixed base. Furthermore, large vertical deflections are observed before the rotor reaches its critical speeds; they are mainly due to combination of base motion and proper vibrations as shown by the spectrogram. The horizontal deflections are also influenced by base motion. Referring to the spectrogram in Figure 17c, we can see that the second FW natural frequency is excited by the base movement $(200 \mathrm{~Hz})$ in the vicinity of $t=3 \mathrm{~s}$. Although the base movement is vertical, growing horizontal deflections are observed owing to gyroscopic coupling.

\section{Conclusion}

In this work, a finite element model is developed to simulate the dynamic behavior of a rotor on a moving rigid base, running at variable spin speed. The movement of the base is general ( 3 translations and 3 rotations). To validate the model, simulations of particular cases for constant spin speed are presented and compared with other works $[7,11]$. A complete concordance is observed.

Furthermore, simulations of the dynamic behavior of on-board rotors with variable spin speed in different situations of base motion are presented and discussed. For linear variation of spin speed, in the cases of sinusoidal rotation or sinusoidal vertical translation of the base, it can be noted in the frequency rage considered, the existence of two resonances: the first one is excited by mass unbalance and it always occurs at the same time, whereas the second one is excited by the base motion and is changing its position in time axis depending on the base frequency.

For exponential variation of spin speed, the deflection graphs show peaks corresponding to backward and forward critical speeds excited by mass unbalance. Furthermore, large vertical deflections are observed before the 
(a)
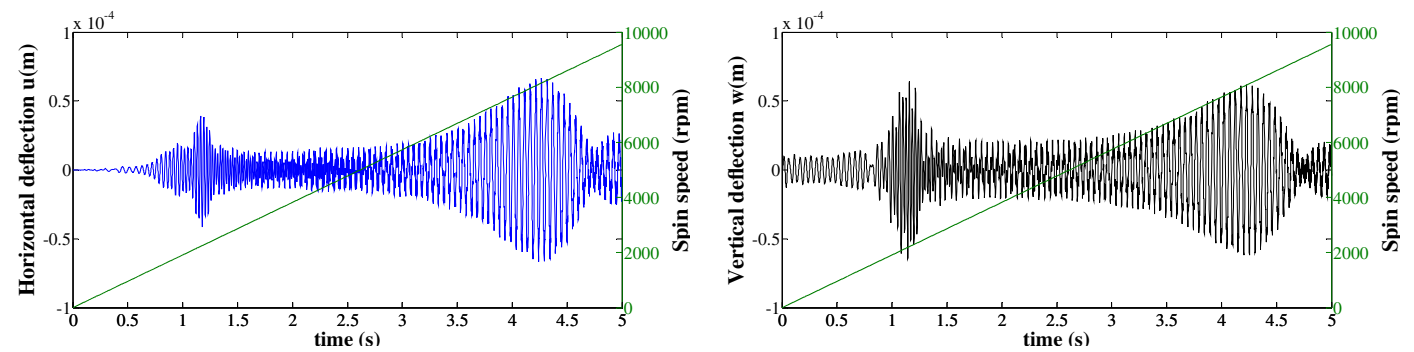

(b)
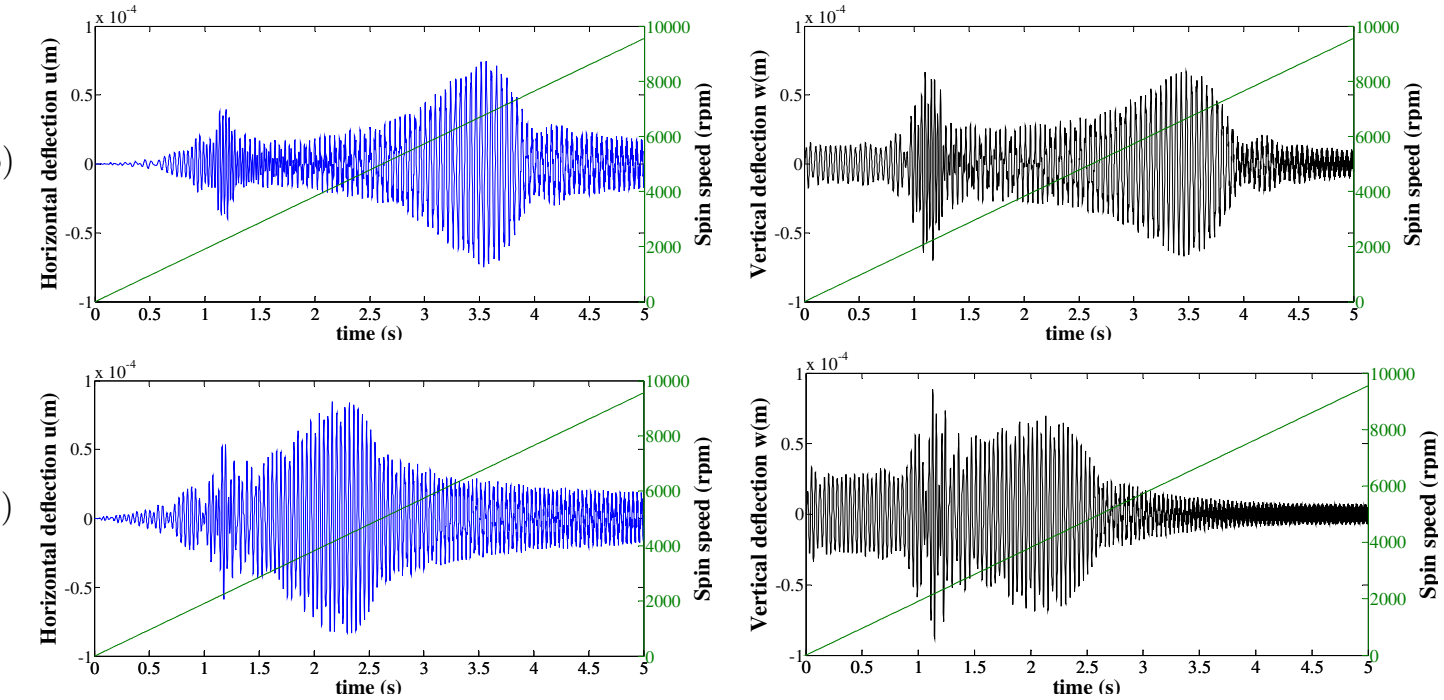

Fig. 12. Linear spin speed of the rotor $\dot{\phi}(t)=200 t \mathrm{rd} . \mathrm{s}^{-1}$ with sinusoidal vertical translation of the base of amplitude $1.5 \times 10^{-5} \mathrm{~m}$ with different frequencies : (a) $20 \mathrm{~Hz}$; (b) $22 \mathrm{~Hz}$; (c) $26 \mathrm{~Hz}$.

rotor reaches its critical speeds; they are due to combination of base motion and proper vibrations. Furthermore, the second mode natural frequency is excited by the base motion, causing growing horizontal deflections induced by gyroscopic coupling.

The code developed in this work is thus, an important tool for further investigations on on-board rotors running with arbitrarily varying spin speed and subject to any combinations of base motions.

\section{Appendix}

The matrices and vectors used in the equations of the disk (9) are presented below:

$$
\begin{aligned}
& {\left[\mathbf{M}_{d}\right]=\left[\begin{array}{cccc}
m_{d} & 0 & 0 & 0 \\
0 & m_{d} & 0 & 0 \\
0 & 0 & I_{d} & 0 \\
0 & 0 & 0 & I_{d}
\end{array}\right]}
\end{aligned}
$$

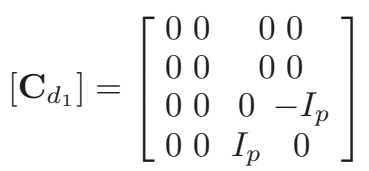

$$
\begin{aligned}
& {\left[\mathbf{C}_{d_{2}}\right]=\left[\begin{array}{cccc}
0 & 2 m_{d} & 0 & 0 \\
-2 m_{d} & 0 & 0 & 0 \\
0 & 0 & 0 & -2 I_{d} I_{p} \\
0 & 0 & -2 I_{d}+I_{p} & 0
\end{array}\right]}
\end{aligned}
$$

The parameters used in the expression of $\left\{\mathbf{F}_{d}\right\}$ are given below:

$$
\begin{aligned}
\lambda_{1} & =\ddot{x}_{b}-2 \dot{\Gamma}_{z} \dot{y}_{b}+2 \dot{\Gamma}_{y} \dot{z}_{b}-\left(\dot{\Gamma}_{y}^{2}+\dot{\Gamma}_{z}^{2}\right) x_{b}+\left(\dot{\Gamma}_{x} \dot{\Gamma}_{y}-\ddot{\Gamma}_{z}\right) y_{b} \\
& +\left(\dot{\Gamma}_{x} \dot{\Gamma}_{z}+\ddot{\Gamma}_{y}\right) z_{b} \\
\lambda_{2} & =\ddot{z}_{b}-2 \dot{\Gamma}_{y} \dot{x}_{b}+2 \dot{\Gamma}_{x} \dot{y}_{b}-\left(\dot{\Gamma}_{x}^{2}+\dot{\Gamma}_{y}^{2}\right) z_{b}+\left(\dot{\Gamma}_{x} \dot{\Gamma}_{z}-\ddot{\Gamma}_{y}\right) x_{b} \\
& +\left(\dot{\Gamma}_{y} \dot{\Gamma}_{z}+\ddot{\Gamma}_{x}\right) y_{b} \\
\lambda_{3} & =\dot{\Gamma}_{x} \dot{\Gamma}_{y}-\ddot{\Gamma}_{z} \\
\lambda_{4} & =\dot{\Gamma}_{y} \dot{\Gamma}_{z}+\ddot{\Gamma}_{x}
\end{aligned}
$$

The matrices and vectors used in the equations of the shaft (17)-(20) are presented below

$$
\begin{gathered}
{\left[\mathbf{M}_{1}\right]=\frac{\rho A L}{420}} \\
\left.\times \begin{array}{cccccccc}
156 & 0 & 0 & -22 L & 54 & 0 & 0 & 13 L \\
0 & 156 & 22 L & 0 & 0 & 54 & -13 L & 0 \\
0 & 22 L & 4 L^{2} & 0 & 0 & 13 L & -3 L^{2} & 0 \\
-22 L & 0 & 0 & 4 L^{2} & -13 L & 0 & 0 & -3 L^{2} \\
54 & 0 & 0 & -13 L & 156 & 0 & 0 & 22 L \\
0 & 54 & 13 L & 0 & 0 & 156 & -22 L & 0 \\
0 & -13 L & -3 L^{2} & 0 & 0 & -22 L & 4 L^{2} & 0 \\
13 L & 0 & 0 & -3 L^{2} & 22 L & 0 & 0 & 4 L^{2}
\end{array}\right]
\end{gathered}
$$


R. Bouziani and N. Ouelaa: Mechanics \& Industry 18, 308 (2017)

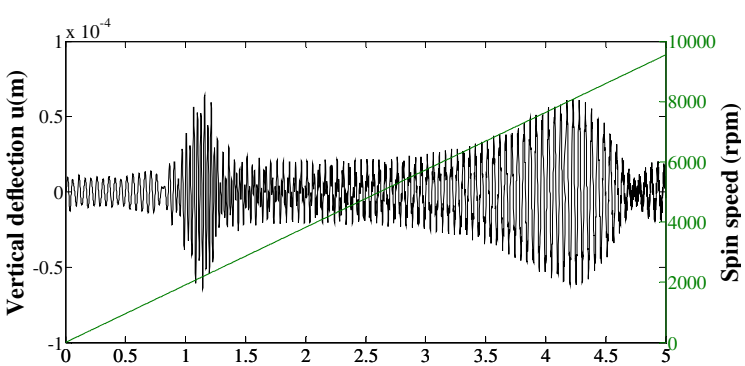

(a)

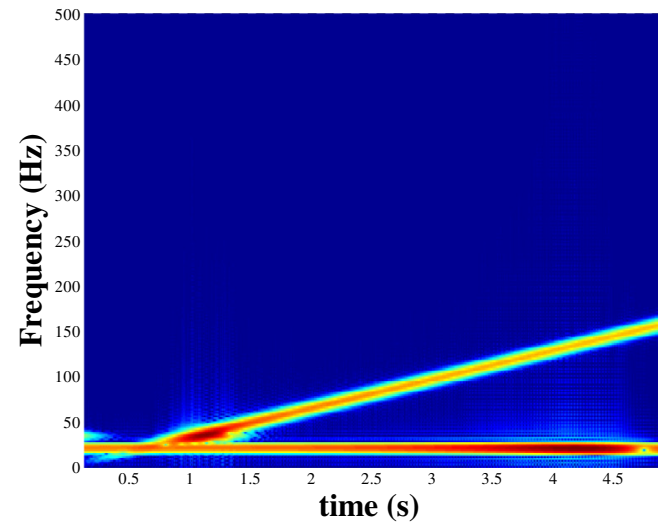

(b)

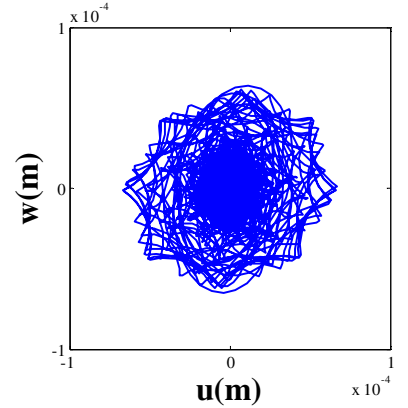

(c)

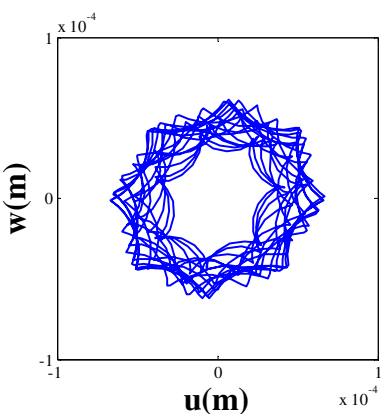

$(\mathrm{d})$

Fig. 13. Linear spin speed of a rotor with vertical sinusoidal translation of the base with a frequency of $20 \mathrm{~Hz}$. (a) Vertical deflection $w$. (b) Spectrogram corresponding to $w$. (c) Orbit. (d) Orbit from 3.7 s to $4.5 \mathrm{~s}$.

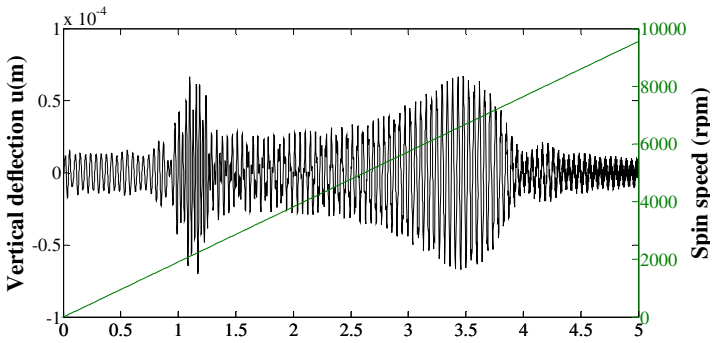

(a)

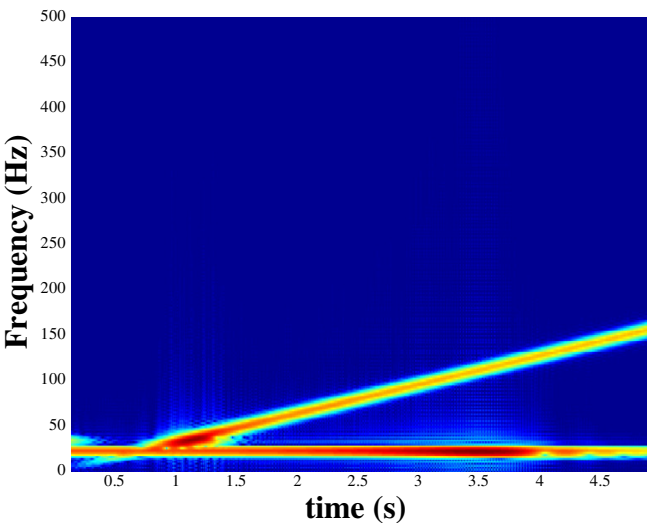

(b)

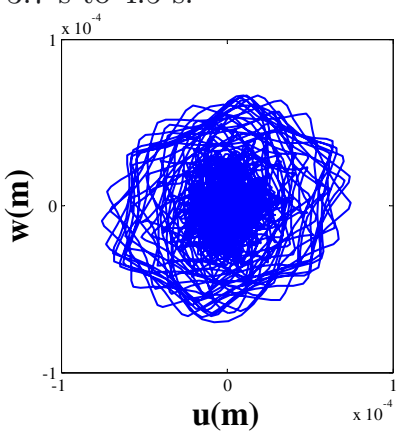

(c)

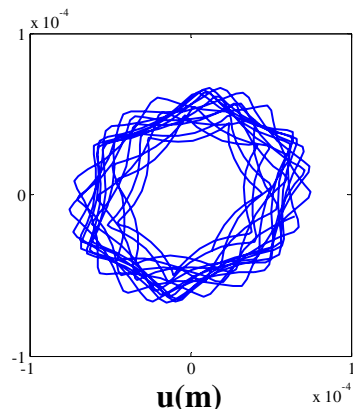

(d)

Fig. 14. Linear spin speed of a rotor with vertical sinusoidal translation of the base with a frequency of $22 \mathrm{~Hz}$. (a) Vertical deflection $w$. (b) Spectrogram corresponding to $w$. (c) Orbit. (d) Orbit from 3 s to 3.7 s. 


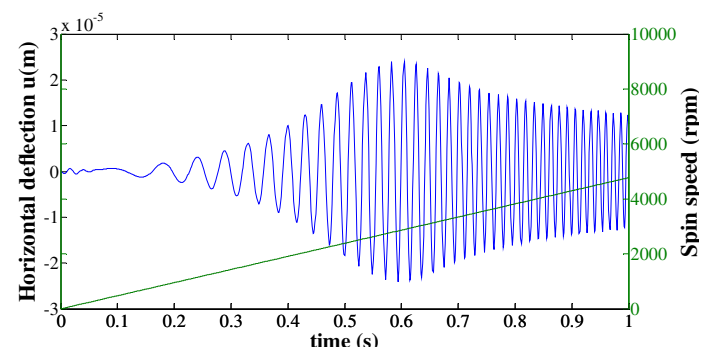

(a)

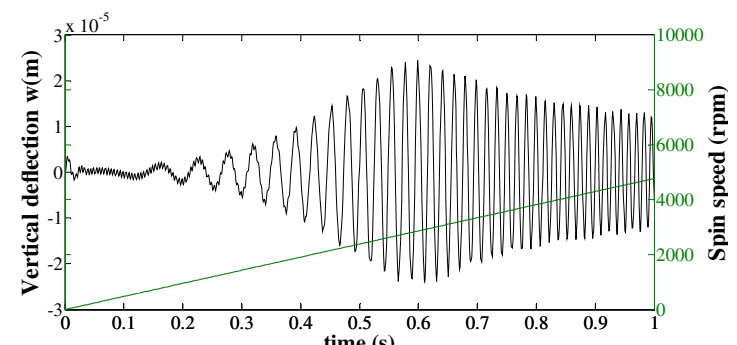

(b)

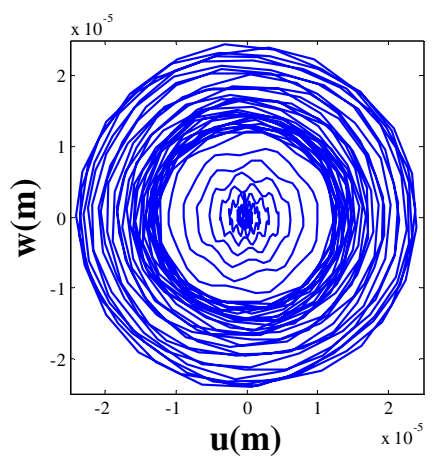

(c)

Fig. 15. Linear spin speed of a rotor with a combined movement of the base: vertical sinusoidal translation with a constant angular velocity about the rotor axis. (a) Horizontal deflection. (b) Vertical deflection. (c) Orbit.
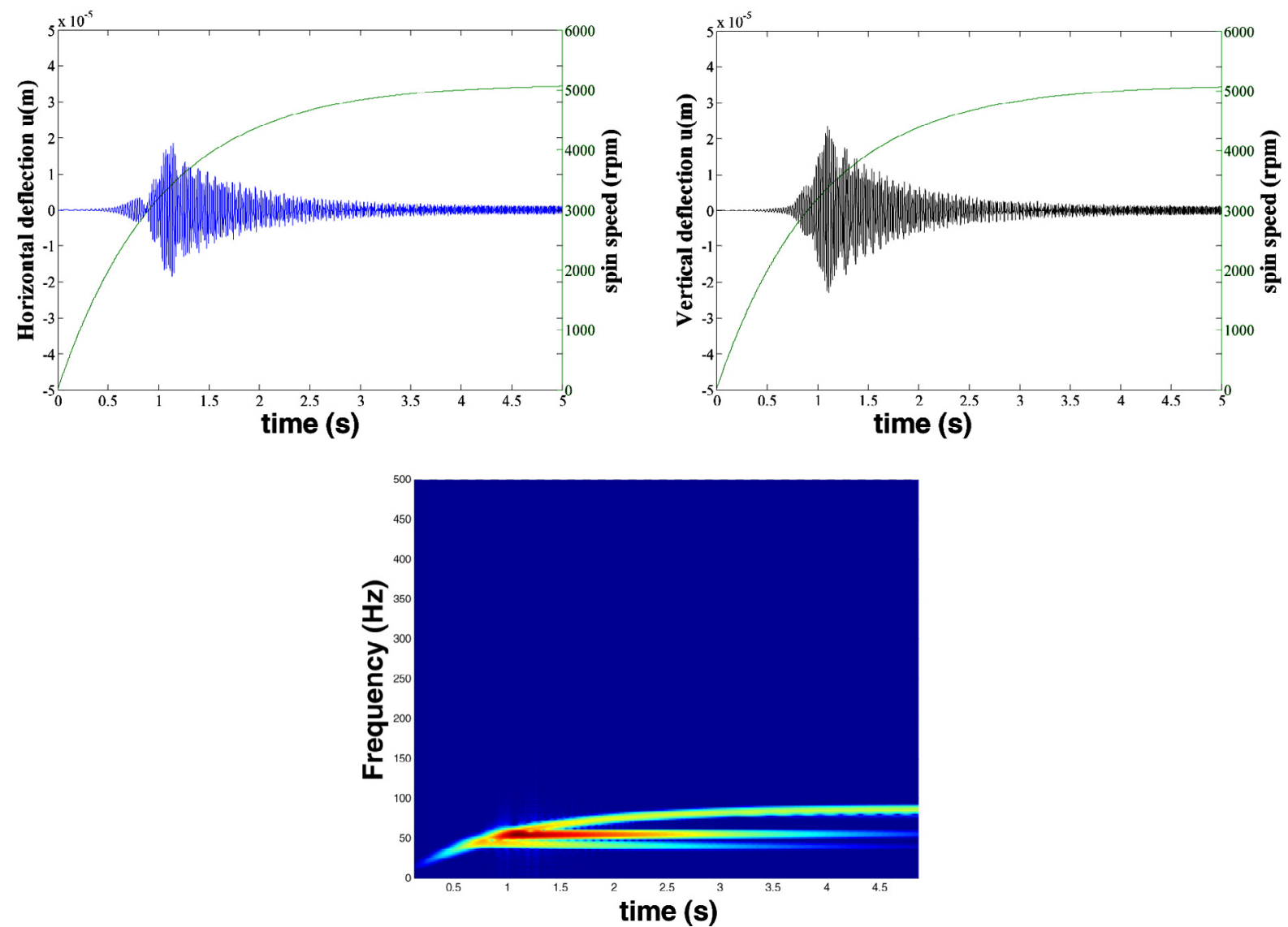

Fig. 16. Response to exponential spin speed of a rotor on a fixed base: displacements and spectrogram corresponding to vertical deflection. 


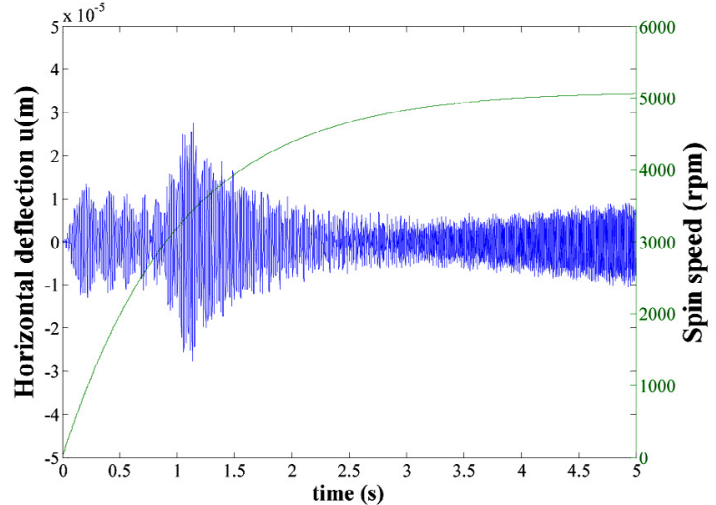

(a)

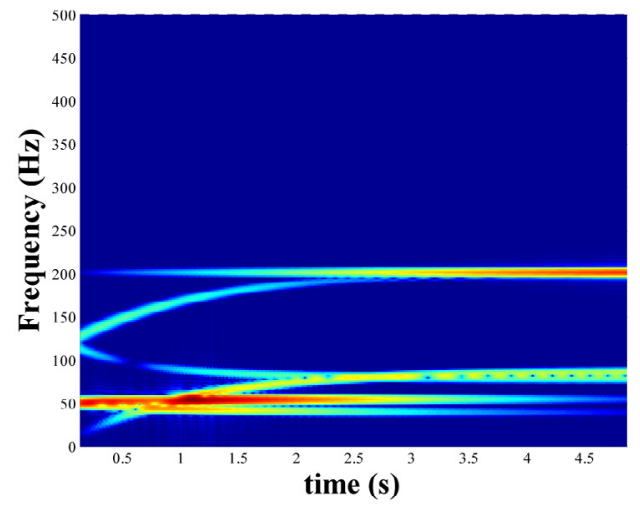

(c)

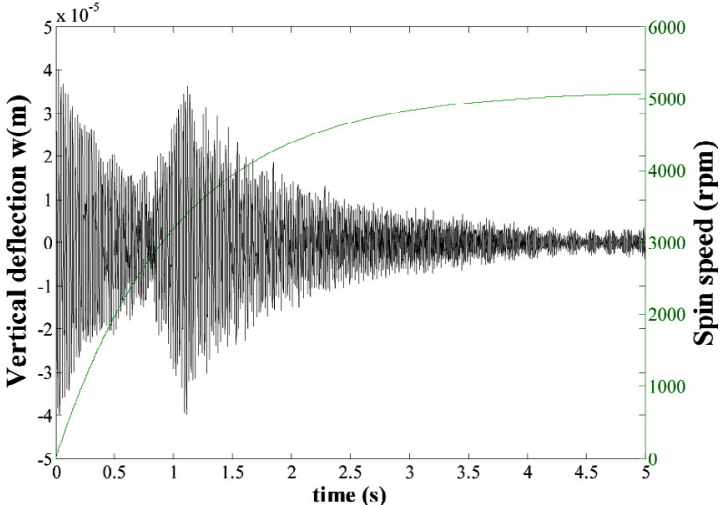

(b)

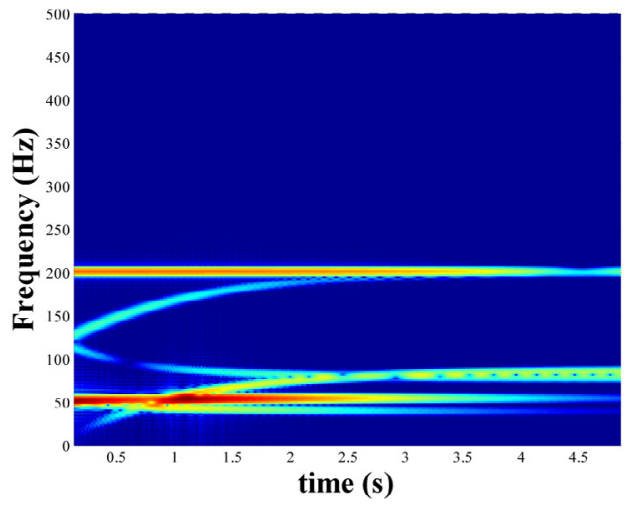

(d)

Fig. 17. Response to exponential spin speed of a rotor mounted on a base subject to sinusoidal translational motion: (a) Horizontal deflection $u$, (b) vertical deflection $w$, (c) spectrogram corresponding to $u$ (d) spectrogram corresponding to $w$.

$$
\begin{aligned}
& {\left[\mathbf{M}_{s 1}\right]=\frac{\rho I}{30 L}} \\
& \times\left[\begin{array}{cccccccc}
36 & 0 & 0 & -3 L & -36 & 0 & 0 & -3 L \\
0 & 36 & 3 L & 0 & 0 & -36 & 3 L & 0 \\
0 & 3 L & 4 L^{2} & 0 & 0 & -3 L & -L^{2} & 0 \\
-3 L & 0 & 0 & 4 L^{2} & 3 L & 0 & 0 & -L^{2} \\
-36 & 0 & 0 & 3 L & 36 & 0 & 0 & 3 L \\
0 & -36 & -3 L & 0 & 0 & 36 & -3 L & 0 \\
0 & 3 L & -L^{2} & 0 & 0 & -3 L & 4 L^{2} & 0 \\
-3 L & 0 & 0 & -L^{2} & 3 L & 0 & 0 & 4 L^{2}
\end{array}\right]
\end{aligned}
$$

$$
\begin{gathered}
{\left[\mathbf{C}_{2}\right]=\frac{\rho A L}{420}} \\
\times\left[\begin{array}{cccccccc}
0 & 156 & 22 L & 0 & 0 & 54 & -13 L & 0 \\
-156 & 0 & 0 & 22 L & -54 & 0 & 0 & -13 L \\
-22 L & 0 & 0 & 4 L^{2} & -13 L & 0 & 0 & -3 L^{2} \\
0 & -22 L & -4 L^{2} & 0 & 0 & -13 L & 3 L^{2} & 0 \\
0 & 54 & 13 L & 0 & 0 & 156 & -22 L & 0 \\
-54 & 0 & 0 & 13 L & -156 & 0 & 0 & -22 L \\
13 L & 0 & 0 & -3 L^{2} & 22 L & 0 & 0 & 4 L^{2} \\
0 & 13 L & 3 L^{2} & 0 & 0 & 22 L & -4 L^{2} & 0
\end{array}\right]
\end{gathered}
$$

$$
\left[\mathbf{K}_{1}\right]=\dot{\Gamma}_{x}^{2}\left[\mathbf{K}_{11}\right]-\dot{\Gamma}_{y}^{2}\left[\mathbf{M}_{1}\right]+\dot{\Gamma}_{z}^{2}\left[\mathbf{K}_{13}\right]
$$

$$
\begin{aligned}
& {\left[\mathbf{C}_{1}\right]=\frac{\rho I}{30 L}} \\
& \times\left[\begin{array}{cccccccc}
0 & -36 & -3 L & 0 & 0 & 36 & -3 L & 0 \\
36 & 0 & 0 & -3 L & -36 & 0 & 0 & -3 L \\
3 L & 0 & 0 & -4 L^{2} & -3 L & 0 & 0 & -L^{2} \\
0 & 3 L & 4 L^{2} & 0 & 0 & -3 L & -L^{2} & 0 \\
0 & 36 & 3 L & 0 & 0 & -36 & 3 L & 0 \\
-36 & 0 & 0 & 3 L & 36 & 0 & 0 & 3 L \\
3 L & 0 & 0 & L^{2} & -3 L & 0 & 0 & -4 L^{2} \\
0 & 3 L & -L^{2} & 0 & 0 & -3 L & 4 L^{2} & 0
\end{array}\right]
\end{aligned}
$$




$$
\begin{aligned}
& {\left[\mathbf{K}_{13}\right]=\frac{\rho A L}{420}\left[\begin{array}{cccccccc}
-156 & 0 & 0 & 22 L & -54 & 0 & 0 & -13 L \\
0 & 0 & 0 & 0 & 0 & 0 & 0 & 0 \\
0 & 0 & 0 & 0 & 0 & 0 & 0 & 0 \\
22 L & 0 & 0 & -4 L^{2} & 13 L & 0 & 0 & 3 L^{2} \\
-54 & 0 & 0 & 13 L & -156 & 0 & 0 & -22 L \\
0 & 0 & 0 & 0 & 0 & 0 & 0 & 0 \\
0 & 0 & 0 & 0 & 0 & 0 & 0 & 0 \\
-13 L & 0 & 0 & 3 L^{2} & -22 L & 0 & 0 & -4 L^{2}
\end{array}\right]} \\
& {\left[\mathbf{K}_{2}\right]=\frac{\rho A L}{420}} \\
& \times\left[\begin{array}{cccccccc}
0 & 156 & 22 L & 0 & 0 & 54 & -13 L & 0 \\
156 & 0 & 0 & -22 L & 54 & 0 & 0 & 13 L \\
22 L & 0 & 0 & -4 L^{2} & 13 L & 0 & 0 & 3 L^{2} \\
0 & -22 L & -4 L^{2} & 0 & 0 & -13 L & 3 L^{2} & 0 \\
0 & 54 & 13 L & 0 & 0 & 156 & -22 L & 0 \\
54 & 0 & 0 & -13 L & 156 & 0 & 0 & 22 L \\
-13 L & 0 & 0 & 3 L^{2} & -22 L & 0 & 0 & -4 L^{2} \\
0 & 13 L & 3 L^{2} & 0 & 0 & 22 L & -4 L^{2} & 0
\end{array}\right] \\
& {\left[\mathbf{K}_{3}\right]=\dot{\Gamma}_{x}^{2}\left[\mathbf{K}_{31}\right]+\dot{\Gamma}_{y}^{2}\left[\mathbf{M}_{s 1}\right]+\dot{\Gamma}_{z}^{2}\left[\mathbf{K}_{33}\right]} \\
& {\left[\mathbf{K}_{31}\right]=\frac{\rho I}{30 L}\left[\begin{array}{cccccccc}
36 & 0 & 0 & -3 L & -36 & 0 & 0 & -3 L \\
0 & 0 & 0 & 0 & 0 & 0 & 0 & 0 \\
0 & 0 & 0 & 0 & 0 & 0 & 0 & 0 \\
-3 L & 0 & 0 & 4 L^{2} & 3 L & 0 & 0 & -L^{2} \\
-36 & 0 & 0 & 3 L & 36 & 0 & 0 & 3 L \\
0 & 0 & 0 & 0 & 0 & 0 & 0 & 0 \\
0 & 0 & 0 & 0 & 0 & 0 & 0 & 0 \\
-3 L & 0 & 0 & -L^{2} & 3 L & 0 & 0 & 4 L^{2}
\end{array}\right]} \\
& {\left[\mathbf{K}_{33}\right]=\frac{\rho I}{30 L}\left[\begin{array}{cccccccc}
0 & 0 & 0 & 0 & 0 & 0 & 0 & 0 \\
0 & 36 & 3 L & 0 & 0 & -36 & 3 L & 0 \\
0 & 3 L & 4 L^{2} & 0 & 0 & -3 L & -L^{2} & 0 \\
0 & 0 & 0 & 0 & 0 & 0 & 0 & 0 \\
0 & 0 & 0 & 0 & 0 & 0 & 0 & 0 \\
0 & -36 & -3 L & 0 & 0 & 36 & -3 L & 0 \\
0 & 3 L & -L^{2} & 0 & 0 & -3 L & 4 L^{2} & 0 \\
0 & 0 & 0 & 0 & 0 & 0 & 0 & 0
\end{array}\right]} \\
& {\left[\mathbf{K}_{4}\right]=\frac{\rho I}{30 L}} \\
& \times\left[\begin{array}{cccccccc}
0 & 36 & 3 L & 0 & 0 & -36 & 3 L & 0 \\
36 & 0 & 0 & -3 L & -36 & 0 & 0 & -3 L \\
3 L & 0 & 0 & -4 L^{2} & -3 L & 0 & 0 & L^{2} \\
0 & -3 L & -4 L^{2} & 0 & 0 & 3 L & L^{2} & 0 \\
0 & -36 & -3 L & 0 & 0 & 36 & -3 L & 0 \\
-36 & 0 & 0 & 3 L & 36 & 0 & 0 & 3 L \\
3 L & 0 & 0 & L^{2} & -3 L & 0 & 0 & -4 L^{2} \\
0 & -3 L & L^{2} & 0 & 0 & 3 L & -4 L^{2} & 0
\end{array}\right]
\end{aligned}
$$

$$
\left[\mathbf{K}_{5}\right]=-\frac{2 \rho I}{30 L}\left[\begin{array}{cccccccc}
0 & 36 & 3 L & 0 & 0 & -36 & 3 L & 0 \\
0 & 0 & 0 & 0 & 0 & 0 & 0 & 0 \\
0 & 0 & 0 & 0 & 0 & 0 & 0 & 0 \\
0 & -3 L & -4 L^{2} & 0 & 0 & 3 L & L^{2} & 0 \\
0 & -36 & -3 L & 0 & 0 & 36 & -3 L & 0 \\
0 & 0 & 0 & 0 & 0 & 0 & 0 & 0 \\
0 & 0 & 0 & 0 & 0 & 0 & 0 & 0 \\
0 & -3 L & L^{2} & 0 & 0 & 3 L & -4 L^{2} & 0
\end{array}\right]
$$

$$
\left[\mathbf{K}_{s 1}\right]=\frac{E I}{L^{3}}\left[\begin{array}{cccccccc}
12 & 0 & 0 & -6 L & -12 & 0 & 0 & -6 L \\
0 & 12 & 6 L & 0 & 0 & -12 & 6 L & 0 \\
0 & 6 L & 4 L^{2} & 0 & 0 & -6 L & 2 L^{2} & 0 \\
-6 L & 0 & 0 & 4 L^{2} & 6 L & 0 & 0 & 2 L^{2} \\
-12 & 0 & 0 & 6 L & 12 & 0 & 0 & 6 L \\
0 & -12 & -6 L & 0 & 0 & 12 & -6 L & 0 \\
0 & 6 L & 2 L^{2} & 0 & 0 & -6 L & 4 L^{2} & 0 \\
-6 L & 0 & 0 & 2 L^{2} & 6 L & 0 & 0 & 4 L^{2}
\end{array}\right]
$$

$$
\left\{\mathbf{F}_{s}\right\}=-\rho A\left\{\begin{array}{c}
\frac{L}{2} \lambda_{1}+\frac{3 L^{2}}{20} \lambda_{3} \\
\frac{L}{2} \lambda_{2}+\frac{3 L^{2}}{20} \lambda_{4} \\
\frac{L^{2}}{12} \lambda_{2}+\frac{L^{3}}{30} \lambda_{4} \\
-\frac{L^{2}}{12} \lambda_{1}-\frac{L^{3}}{30} \lambda_{3} \\
\frac{L}{2} \lambda_{1}+\frac{7 L^{2}}{20} \lambda_{3} \\
\frac{L}{2} \lambda_{2}+\frac{7 L^{2}}{20} \lambda_{4} \\
-\frac{L^{2}}{12} \lambda_{2}-\frac{L^{3}}{20} \lambda_{4} \\
\frac{L^{2}}{12} \lambda_{1}+\frac{L^{3}}{20} \lambda_{3}
\end{array}\right\}\left\{\begin{array}{c}
2 \dot{\Gamma}_{x}\left(\dot{\Gamma}_{y}+\dot{\phi}\right)-\lambda_{3} \\
2 \dot{\Gamma}_{z}\left(\dot{\Gamma}_{y}+\dot{\phi}\right)-\lambda_{4} \\
0 \\
0 \\
-2 \dot{\Gamma}_{x}\left(\dot{\Gamma}_{y}+\dot{\phi}\right)+\lambda_{3} \\
-2 \dot{\Gamma}_{z}\left(\dot{\Gamma}_{y}+\dot{\phi}\right)+\lambda_{4} \\
0 \\
0 \\
0
\end{array}\right\}
$$

\section{References}

[1] M. Lalanne, G. Ferraris, Rotordynamics prediction in engineering, John Wiley, New York, 1998

[2] G. Genta, Dynamics of Rotating Systems, Springer, New York, 2005

[3] M.I. Friswell, J.E.T. Penny, S.D. Garvey, A.W. Lees, Dynamics of Rotating Machines, Cambridge University Press, 2010 
[4] F. Lin, G. Meng, Study on the dynamics of a rotor in a manoeuvring aircraft, Trans. ASME J. Vib. Acoust. 125 (2003) 324-327

[5] M. Duchemin, Contribution à l'étude du comportement dynamique d'un rotor embarqué, Doctorat Thesis, INSALyon, 2003

[6] M. Duchemin, A. Berlioz, G. Ferraris, Dynamic Behavior and Stability of a Rotor under Base Excitations, J. Vib. Acoustics 128 (2006) 576-585

[7] N. Driot, C.H. Lamarque, A. Berlioz, Theoretical and Experimental Analysis of a Base Excited Rotor, ASME Journal of Computational and Nonlinear Dynamics, Trans. ASME 1 (2006) 257-263

[8] A.S. Das, J.K. Dutt, K. Ray, Active vibration control of unbalanced flexible rotor-shaft systems parametrically excited due to base motion, Appl. Mathematical Modelling 34 (2010) 2353-2369

[9] A.S. Lee, B.O. Kim, Y.-C. Kim, finite element transient response analysis method of a rotor bearing system to base shock excitations using the state-space Newmark scheme and comparisons with experiments, J. Sound Vib. 297 (2006) 595-615

[10] F.M.A El-Saeidy, F. Sticher, Dynamics of a rigid rotor linear/nonlinear bearings system subject to rotating unbalance and base excitations, J. Vib. Control 16 (2010) 403-438
[11] M. Dakel, S. Baguet, R. Dufour, Steady state dynamic behavior of an on-board rotor under combined base motions, Journal of Vibration and Control, November 2014 20: 2254-2287, first published on June 24, 2013

[12] M. Dakel, S. Baguet, R. Dufour, Nonlinear dynamics of a support-excited flexible rotor with hydrodynamic journal bearings, J. Sound Vib. (2014)

[13] Q. Han, F. Chu, Dynamic response of cracked rotorbearing system under time-dependent base movements, J. Sound Vib. 33 (2013) 6847-6870

[14] Q. Han, F. Chu, Dynamic behaviors of a geared rotor system under time-periodic base angular motions, Mechanism and Machine Theory 78 (2014) 1-14

[15] Q. Han, F. Chu, Parametric instability of flexible rotorbearing system under time-periodic base angular motions, Applied Mathematical Modelling, 39 (2015) 45114522

[16] H. Irretier, Vibrations in Variable Speed Machines, in: CISM Courses and Lectures No 297 : Rotordynamics 2, Problems in Turbomachinery, edited by N.F.Riege, Springer-Verlag Wien New York, 1988, pp. 571-586 